\title{
Stanene the new gas sensing wonder material: current status and future prospects
}

\author{
M.W.Aqra ${ }^{1}$ and A.A. Ramanathan ${ }^{2 *}$ \\ ${ }^{1}$ National Center for Research and Development, Amman-11941, Jordan \\ ${ }^{2}$ Department of Physics, University of Jordan, Amman-11942, Jordan \\ *Corresponding author Email: amallahmad@gmail.com
}

\begin{abstract}
In the search for new 2D materials beyond graphene with similar exemplary properties Stanene the new graphene relative was successfully synthesized and characterized in 2016 [14]. This new material which consists of atomically thin tin ( $\mathrm{Sn}$ ) atoms arranged in a hexagonal lattice has shown great promise in gas sensing applications in its pristine, doped and functionalized forms as evidenced by the recent research outputs following its discovery. Researchers have discovered that with its exotic properties it is highly efficient in the detection of the environmentally harmful gases like $\mathrm{SO}_{2}, \mathrm{NO}_{2}, \mathrm{CO}_{2}, \mathrm{NH}_{3}$ and can serve as a multifunctional gas sensor.

In this focused and up-to-date review we aim to group the literature on first principles studies of stanene regards to its application for gas sensing and critically examine the success/failures of its multifunctional gas sensing properties as compared to graphene. We systematically present and discusses how structural deformations, defects and doping effect the gas sensing performance. Despite the impressive progress in the field of gas sensing with stanene shown by first principles calculations, many challenges in large scale synthesis, device fabrication and commercialization need to be addressed. The current review can help understand the current status and drawbacks of stanene and identify future directions in this field.
\end{abstract}

Key words: 2D materials; toxic gases; doping; chemical functionalization; green house gases 


\section{Introduction}

Gas adsorption and sensing is very important in today's increasingly industrialised world with the presence of so many harmful gases like $\mathrm{SO}_{2}, \mathrm{NO}_{2}, \mathrm{~N}_{2} \mathrm{O}$ and $\mathrm{NH}_{3}$ detrimental to the environment and human well being. Accurate detection of these toxic gases as well as the monitoring of $\mathrm{CO}_{2}, \mathrm{O}_{3}$ and $\mathrm{O}_{2}$ levels in the atmosphere require selective and sensitive sensors; and nanomaterials with their high surface to volume ratio are very attractive offering large surface areas for adsorption. The last two decades has seen a lot activity in finding suitable nanostructured materials for gas sensors and graphene (Gr) and Gr like 2D materials have played a major role in this aspect [1-5].

The exemplary performance of $\mathrm{Gr}$ and its exotic properties and the immense possibilities available with the different nanostructures single, bi and few layers of Gr, CNTs, nano-ribbons and nano-composites for diverse applications in optoelectronics, energy, desalination, sensing [610] and other fields has led to a race to find new Gr analogues. The quest for other elemental analogues of $\mathrm{Gr}$ has resulted in various studies on Antimonene, Arsenene, Bismuthene, Borophene, Germanene, Indiene, Phosphorene, Silicene and Stanene [11-13].

Among these Stanene a 2D hexagonal lattice of Sn (Tin) atoms was successfully synthesized and investigated by Saxena et al in 2016 [14]. They were able to synthesize from mono to few atomic layers of free standing stanene and characterize them optically using UV-Vis absorption, Raman and photoluminescence spectroscopy and prove experimentally its existence and stability. Their VASP DFT first principles calculations suggest that stanene stabilizes in low buckled configuration with buckling height of $\Delta=0.85 \AA$, in good agreement with previously reported data [11]. The buckling causes an overlap of the $\pi$ and $\sigma$ orbitals and weakens the $\pi-\pi$ bonds. Table I lists the physical and electronic properties of Stanene in comparison with Gr.

Table 1.Summary of the physical and electronic properties of Stanene and graphene

\begin{tabular}{|c|c|c|c|c|}
\hline $\begin{array}{l}\text { Physical/Electronic } \\
\text { property }\end{array}$ & Stanene & Ref & Graphene & Ref \\
\hline Structure & $\begin{array}{l}\text { Buckled atomic layer of } \mathrm{Sn} \text { atoms in } \\
\text { hexagonal honeycomb lattice }\end{array}$ & $\begin{array}{l}{[11],} \\
{[14]}\end{array}$ & $\begin{array}{l}\text { Single planar atomic layer of } \mathrm{C} \\
\text { atoms in hexagonal honeycomb } \\
\text { lattice }\end{array}$ & {$[15]$} \\
\hline Bonding & $\begin{array}{l}\mathrm{Sp}^{2} \text { and } \mathrm{sp}^{3} ; \\
\text { weak } \pi-\pi \text { bonding }\end{array}$ & {$[11]$} & strong covalent $\mathrm{sp}^{2}$ bonds; & {$[16]$} \\
\hline Band gap & $\begin{array}{l}73 \mathrm{meV} \text { with } \mathrm{SO} ; \text { Topological } \\
\text { insulator }\end{array}$ & {$[17]$} & Gapless semiconductor & {$[16]$} \\
\hline Spin orbit coupling & Strong & & weak & \\
\hline Dielectric constant & $\begin{array}{l}\varepsilon \perp \text { and } \varepsilon \| \text { are } \sim 1.6 \text { and } \sim 14.4 \\
\text { respectively }\end{array}$ & {$[18]$} & $\begin{array}{l}\varepsilon \perp \text { and } \varepsilon \| \text { are } \sim 3 \text { and } \sim 1.8 \\
\text { respectively } @ \text { low E fields }\end{array}$ & {$[19]$} \\
\hline Mobility & $\begin{array}{l}1297.24 \text { and } 1591.53 \mathrm{~cm} / \mathrm{Vs} \text { in the } \mathrm{x} \\
\text { and } y \text { directions. }\end{array}$ & {$[20]$} & $15,000-200,000 \mathrm{~cm} / \mathrm{Vs}$ & {$[16]$} \\
\hline Conductivity & 100\%@room temperature & {$[21]$} & $<100 \%$ & {$[22]$} \\
\hline $\begin{array}{l}\text { Thermal } \\
\text { conductivity }\end{array}$ & $11.6 \mathrm{~W} / \mathrm{mK}$ & {$[23]$} & Up to $4000 \mathrm{~W} / \mathrm{mK}$ & {$[24]$} \\
\hline Mechanical strength & $25.2 \mathrm{GPa}$ & {$[25]$} & $130 \mathrm{GPa}$ & {$[26]$} \\
\hline
\end{tabular}


As seen from the Table, the room temperature topological insulator stanene is a novel 2D material that can overcome the deficiencies of Gr. The excellent electronic properties makes stanene stand out with its exceptional gas sensing performance as shown by the recent investigations of this material by various groups in its pristine, structurally defected and doped forms [27-32]. Stanene appears to be the new 'gas sensing wonder' and it is time to review and recapture the major achievements and progress made in view of the highly intensive theoretical investigations in this topic during the past five years.

This review therefore aims to discuss the latest advancements from the literature survey of the recent first principles studies of stanene regards to its application for gas sensing. We first elaborate the synthesis methods followed by gas-sensing mechanisms used and introduce the various types of gas-sensing devices. We then describe the structural parameters and other factors like defects and doping that influence the gas sensing performance. The current status and drawbacks of stanene for sensing of $\mathrm{SO}_{2}, \mathrm{CO}, \mathrm{CO}_{2}, \mathrm{NO}_{2}, \mathrm{NH}_{3}$ and other harmful gases is examined and compared with graphene. Finally the future prospects and challenges of this cousin of $\mathrm{Gr}$ for gas-sensing applications is presented. The review is arranged according to the main sections: 1. Introduction; 2. Methods for synthesis; 3.Sensing mechanisms; 4.Gas sensing functionalization; 5. Performance; and 6. Challenges and Future outlook.

\section{Synthesis methods}

The fascinating predicted properties of stanene as a 2D topological insulator and its excellent gas sensing capabilities is a stimulus for the synthesis of stanene and its derivatives and further experimental investigations. Group-IV monolayers (ML) have been experimentally realized using various experimental techniques and theoretically characterized in the past few years. [3336]. Compelling evidence of graphene like 2D silicon was provided by Vogt et al [33] with the synthesis of epitaxial silicene sheets grown on $\operatorname{Ag}(111)$ substrate and by Fleurence et al [34] on diboron films.

First principles method for characterization of new materials is a powerful and accurate tool for prediction of properties. It can also be used for modeling ideal growth mechanisms and substrates. Guo et al explored in 2016, the potential of Ag (111) surface as an ideal substrate for the epitaxial growth of monolayer (ML) stanene [37]. Using first-principles calculations, they studied the stability of the structure of stanene and the diffusion behavior of Sn adatom on $\operatorname{Ag}(111)$ surface in different epitaxial relations with respect to $\operatorname{Ag}(111)$ surface. Their major findings that the hexagonal structure of stanene ML is well preserved on the $\operatorname{Ag}(111)$ surface and that the height of epitaxial stanene ML is comparable to the step height of the substrate supports the fact that it is possible to achieve large-area growth of stanene. Figure 1 reproduced from their work indicates that the diffusion barrier of Sn atom on $\operatorname{Ag}(111)$ surface is not only low but also uniform, providing excellent conditions for the large-scale epitaxial growth of stanene ML on $\mathrm{Ag}(111)$ surface even at low temperature. 


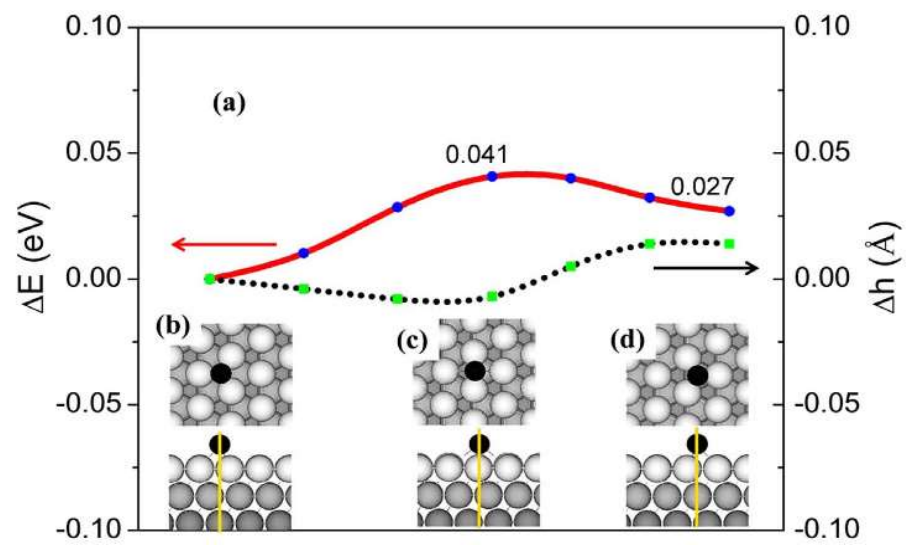

Figure 1. Sn adatom diffusion along a representative path on $\operatorname{Ag}(111)$ surface, $(b-d)$ top view and side view of the Sn diffusion pathway. Energy profiles (red) and height variation (black dash). Both the energy and height of Sn adatom at the FCC position are set to zero. Reproduced with permission from ref. [37]

Once, the epitaxial stanene ML is detached from Ag (111) surface perfect lattice structure of free-standing stanene can be achieved. In addition, their simulated STM images with the height line profiles can provide important references for future experiments. In the recent years 2D buckled films were grown on metals similar to Ag such as germanene on Au [38] and Pt [39], borophene on $\mathrm{Cu}$ [40] and silicene on Ir [41]. These metals could also be good match as substrates for the epitaxial growth of stanene; further verification and experimentation are required.

Recently in 2015, ML and few-layers (FLs) stanene were successfully grown on $\mathrm{Bi}_{2} \mathrm{Te}_{3}$ (111) surface using molecular beam epitaxy (MBE) and the atomic structures and their electronic properties were studied using scanning tunneling microscopy (STM), angle-resolved photoemission spectroscopy (ARPES) and first-principles calculations [42]. It was found that the stanene epitaxial growth on $\mathrm{Bi}_{2} \mathrm{Te}_{3}(111)$ was a mixture of ML, bilayer (BL) and FLs. The reason could be the sharp steps of $\mathrm{Bi}_{2} \mathrm{Te}_{3}(111)$ surface $\sim 1 \mathrm{~nm}$ in height, which are able to block the continuous growth of stanene by suppressing the growth fronts steps. This proves the critical role the substrate plays in the growth mechanism and proper choice of substrate is essential for good epitaxy of stanene ML.

\section{Sensing Mechanisms}

The honeycomb structure of the two dimensional Gr and $\mathrm{Gr}$ analogues gives them exceptional electronic, mechanical, chemical, and physical properties mainly coming from the quantum size effects $[11,43-45]$ as summarized earlier in Table1 for Gr and stanene. A change in the chemical environment by the introduction of a new chemical species or functionalization introduces a change in these properties and can form the basis of detection and sensing mechanisms. In Gr and layered 2D inorganic Gr analogues the main sensing mechanism is based on the charge transfer processes, in which the 2D sensor material acts as charge acceptors or donors. When exposed to different gases, there is a charge transfer process that occurs between the sensor and 
the adsorbed gases, with different magnitudes of charges and direction dependent on the nature of the gas species and 2D sensor interaction.

\subsection{Charge transfer}

The presence of gas/gases changes the chemical environment of the sensor-analyte system results in electronic charge density difference (CDD) due to the charge transfer. A measure of the CDD gives a direct qualitative measure of the gas adsorption. Figure 2 shows an example of a charge transfer mechanism, taken from the work of Yue et al [46] on gas sensing using MoS2 ML, a Gr $2 \mathrm{D}$ analogue. The Gases $\mathrm{O}_{2}, \mathrm{H}_{2} \mathrm{O}, \mathrm{NO}, \mathrm{NO}_{2}$ and $\mathrm{CO}$ all act as charge acceptors as seen from the figure. Only NH3 acts as a charge donor. This same behavior of the gases is what was observed for $\mathrm{Gr}$ and its derivatives and it is also expected with other $\mathrm{Gr}$ analogues sensing materials due the similarities in their structures. In fact this is confirmed in the works of Tang et al [47] for Co anchored Gr and Sv-Gr (Gr with single sulphur vacancy), Abbasi \& Sardroodi [28] for pristine and boron doped stanene and the boron doped stanene work of Garg et al [27].

The charge transfer that takes place during the interaction of the senor material and gas will also change the resistance of the system, and resistance based measurements can also be used to estimate the gas adsorption which is discussed in the next section.

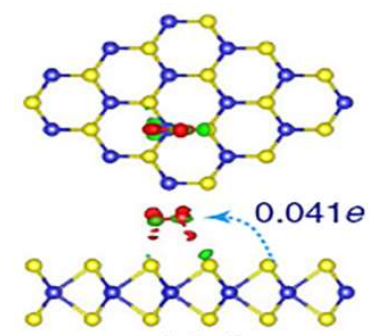

(a) $\mathrm{O}_{2}$

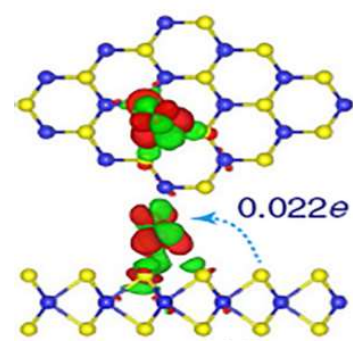

(d) NO

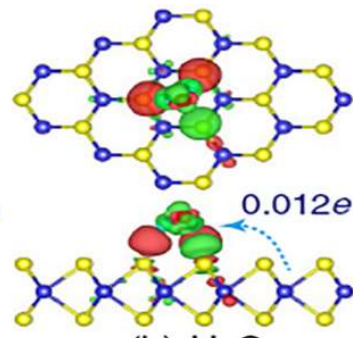

(b) $\mathrm{H}_{2} \mathrm{O}$

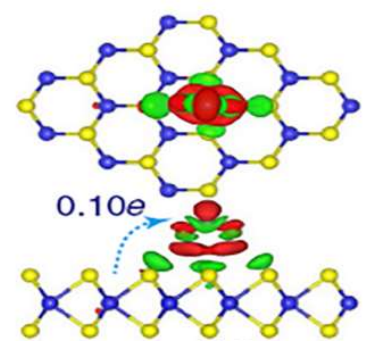

(e) $\mathrm{NO}_{2}$

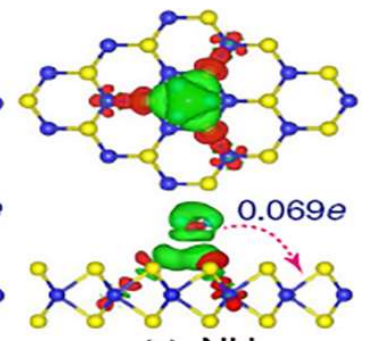

(c) $\mathrm{NH}_{3}$

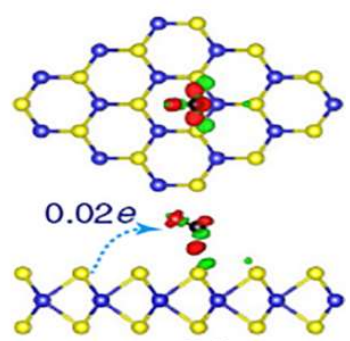

(f) $\mathrm{CO}$

Figure 2.Charge transfer process and density difference plots for (a) O2, (b) $\mathrm{H} 2 \mathrm{O}$, (c) NH3, (d) NO, (e) NO2, and (f) $\mathrm{CO}$ interacting with pristine $\mathrm{ML} \mathrm{MoS}_{2}$. Reproduced with permission from Yue et al., Nanoscale Res. Lett. 8, 425 (2013). Copyright 2013 Springer [46]

\subsection{Resistance based}

Indirect way of estimating the gas adsorption by measuring the change in resistance, or by noting the change in the optical/photoluminescence spectra of the system, which are a result of the electronic charge/spin density change upon the interaction of the $2 \mathrm{D}$ sensor with the gas. 
Resistance based sensor devices could be chemiresistors [48, 49], field effect transistors FET [50, 51], Schottky diode [52], Impedance sensors [53, 54], conductometric semiconducting gas sensors [55-57], Surface acoustic wave (SAW) devices, Surface work function (SWF) [58-60], or heterojunction sensors [61]. The choice of the sensor device is dictated by the user requirements on selectivity, sensitivity, cost, the analyte and other considerations. Chemiresistors are easy to fabricate, simple to use, with low power consumption and ability for reuse; in addition they are cost effective. In comparison FET sensor devices are portable, simple to fabricate, sensitive with nanoscale miniaturization. They can be used to sense many kinds of gases, such as $\mathrm{CO}, \mathrm{NO}, \mathrm{NH} 3, \mathrm{NO} 2, \mathrm{SO} 2, \mathrm{H} 2$ etc [62-64]. Figure 3 shows the schematics and transport characteristics of a FET gas sensor device used for sensing $\mathrm{NO}_{2}$ and $\mathrm{NH}_{3}$ with $\mathrm{rGrO}$ as the conducting channel between the source and drain electrodes, and with $\mathrm{Si}$ as a back-gate electrode reproduced from the work of $\mathrm{Lu}$ et al [65]. The sensor has a response of $100 \mathrm{ppm}$ to $\mathrm{NO}_{2}$ in air.
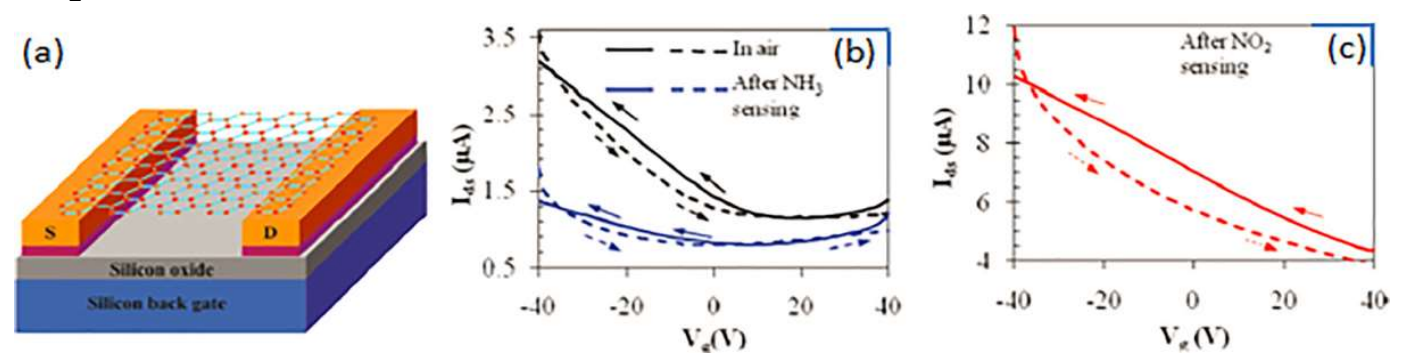

Figure 3.(a) Schematic of the rGO FET sensing device. Transport characteristics (b) before and after an $\mathrm{NH}_{3}$ sensing cycle (c) after an $\mathrm{NO}_{2}$ sensing cycle. Reproduced with permission from Lu et al., ACS Nano 5, 1154 (2011). Copyright 2011 American Chemical Society [65].

Impedance sensors are usually composed of a noble metal, a metal oxide, and layered materials and utilize solid-state impedance spectroscopy to measure the sensing response over a wide range of frequencies from sub-hertz to megahertz. It can accurately detect gases at the one part per million. Low concentrations of various gases including hydrocarbons, $\mathrm{NO}_{\mathrm{x}}, \mathrm{CO}$, and humidity can be detected using these types of gas sensor devices. An impedance gas sensor that selectively detects methanol based on layered black phosphorus and a gold electrode is presented in Figure 4 which is adapted from the work of Mayorga-Martinez et al [66].
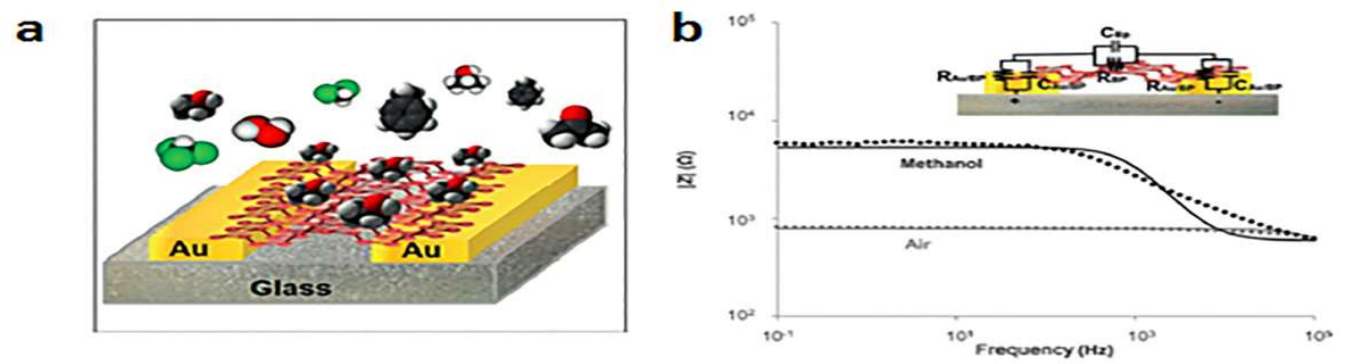

Figure 4.(a) Schematic diagram of the impedance gas sensor based on black phosphorus, (b) the impedance module of black phosphorus in air and in methanol vapor, Reproduced with permission from Mayorga-Martinez et al., Angew. Chem., Int. Ed. 127, 14525 (2015). Copyright 2015 Wiley-VCH Verlag GmbH \& Co. KGaA, Weinheim [66]. 


\section{Gas sensing functionalization}

The sensing performance of 2D materials can be improved by functionalization; these could be physical/structural or chemical. A change in the structure of the material by the application of strain, pressure, temperature or nanostructuring (ML,BL,NTs) will cause a change in the electronic properties of the material that can modify the adsorption characteristics. Another way of modifying the electronic properties is through chemical functionalization i.e. decorating/doping with elements of another species or the introduction of defects/vacancies. All these factors effectively change the electronic charge distribution, density of states (DOS) and band structure of the 2D material and tune the properties to our liking. Figure 5 gives a snapshot of the functionalities that will be discussed and reviewed in relation to the gas sensing capabilities of Gr and stanene.

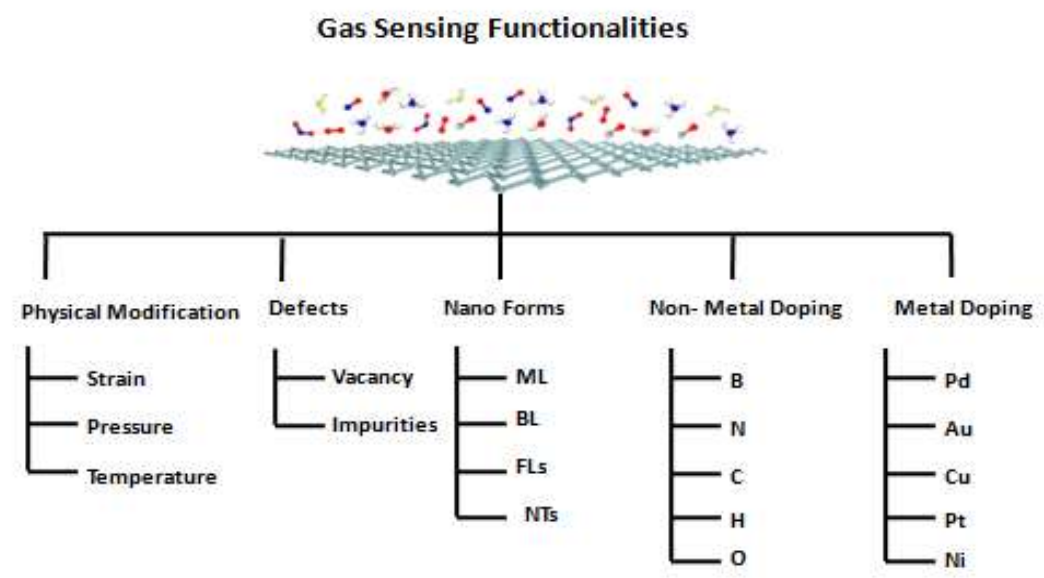

Figure 5.Snapshot of the functionalities for gas sensing with 2D layered materials.

\subsection{Structural deformations (vacancies, defects, strain)}

Strain is an important parameter that will change the structure of a material and causes changes in the bandgap and electronic properties. Modarresi et al. [67] used DFT and molecular mechanics models to study the effect of external strain on stanene with spin orbit coupling (SOC) taken into account. The SOC is important for Sn as it is an heavy metal, and it opens the zero-gap of stanene to a gap of $70 \mathrm{meV}$ and makes it a topological insulator. One of the key issues in high performance FETs operating at room temperature is the presence of a sizeable gap. The sensing performance is therefore enhanced. Upon applying external strain Modarresi and his co-workers observed a closing of this gap and the electronic structure changes that are not favourable for gas sensing. They also studied the size and chirality effects of stanene nanoribbons.

Kumar et al [68] have studied the effect of the role of external defects in the gas sensing capabilities of Gr chemical FETs. They show that the sensitivity (in terms of modulation in 
electrical conductivity) of pristine Gr is actually due to external defects in the insulating substrate which can modulate the electronic properties of Gr. This is shown in Figure 6 reproduced from their graphical abstract.
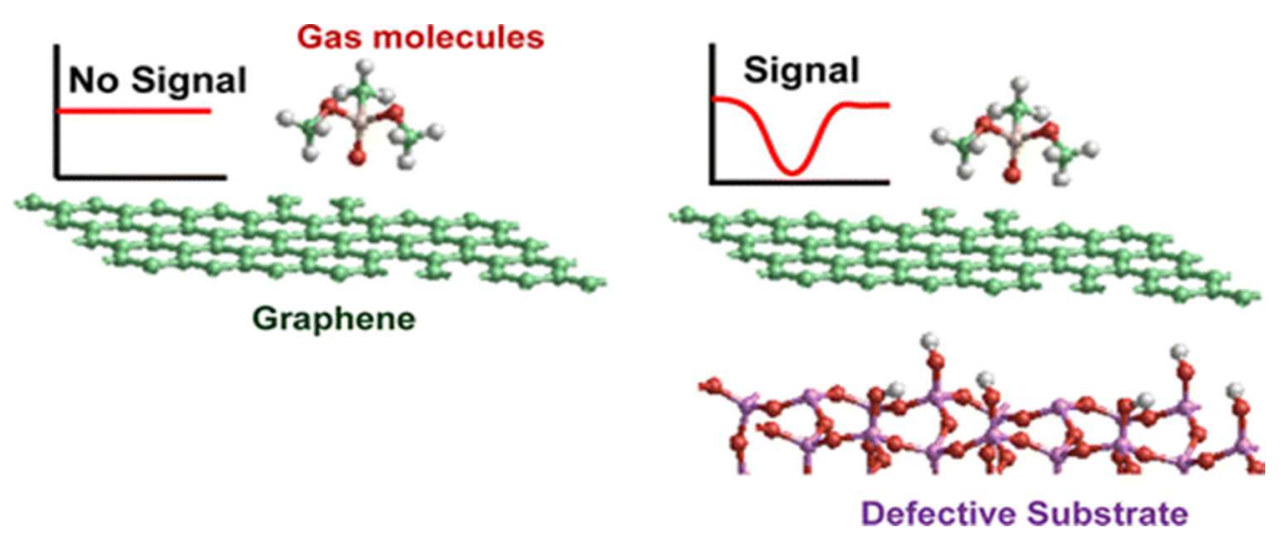

Figure 6.Graphical representation of the effect of defects ofn the FET signals. [68].

\subsection{Non-metal doping}

The effect of non-metals boron and $\mathrm{N}$ doped stanene on gas sensing of NO, NO2, NH3 and N2O have been studied by Garg et al.[27] using DFT and Dispersion corrected (DFT-D3) functionals and spin orbit coupling. Using Bader charge analysis and CDD they show that doping improves the interaction between stanene and gas molecules. They observed that the doped systems (B@-, N@-, and B-N@stanene) show better sensitivity and selectivity than pristine stanene.

Abassi et al [28] also investigated boron doping and examined the adsorption behaviors of triatomic gas molecules $(\mathrm{NO} 2, \mathrm{SO} 2$ and $\mathrm{CO} 2)$ on the buckled stanene nanosheets. Their Mulliken charge and CDD calculations indicated that the adsorption of gas molecules on the doped stanene nanosheets is energetically more favourable than that on the pristine ones in agreement with Garg et al [27].

Although group IV-2D structures silicene, germanene, and stanene have been realized experimentally and have rather high carrier mobilities they are all semimetallic without an appreciable band gap, which makes them unsuitable for FET applications. In an attempt to integrate them in microelectronics recently, Bianco et al [69] successfully fabricated fully hydrogenated germanene (germanane) via a chemical method. Interestingly, germanane is semiconducting with a moderate band gap as well as a considerable carrier mobility greater than $10^{4} \mathrm{~cm}^{2} \mathrm{~V}^{-1} \mathrm{~s}^{-1}$. Hydrogenation seems effective in opening the gap and increasing the gas sensing capabilities. Encouraged by the work of Bianco et al, hybrid density functional theory (DFT) computations were carried out to investigate the structural, electronic, and optical properties of hydrogenated stanene, namely stanene ( $\mathrm{SnH})$ by Liu et al [70]. Their computations revealed that stanane is semiconducting with a direct band gap of $1.00 \mathrm{eV}$ and can be flexibly tuned by applying an external strain. Moreover, they found that stanane has rather strong optical 
absorption in the visible as well as infrared regions of the solar spectrum and electron/hole nobilities higher than that of $\mathrm{MoS}_{2}$.

\subsection{Metal doping}

Stanene has been doped with several metals and transition metals in various first principle studies; Zhang et al investigated the incorporation of transition metals $\mathrm{Ni}, \mathrm{Cu}$ and $\mathrm{Au}$ in stanene ML for gas sensing. They found that the Ni-adsorbed stanene monolayers were semiconductor in nature, while the $\mathrm{Cu}$ and $\mathrm{Au}$-adsorbed ones exhibited metallic property. They examined various adsorption sites and directions for the binding of gas molecules to the Au-adsorbed stanene systems and found that the $\mathrm{Au}$ adsorption enhances the adsorption of gas molecules on the stanene system. Their CDD analysis indicated that the bond in the Au-embedded stanene with adsorbed gas molecules is of a covalent nature due to the location of the favoured electron accumulation sites which are largely at the middle of the Au-gas molecule bond. They predict $\mathrm{Au}$-adsorbed stanene systems as superior candidates for gas sensing.

The interaction of some gas molecules $\left(\mathrm{CO}, \mathrm{NO}, \mathrm{N}_{2} \mathrm{O}\right.$ and $\mathrm{NH}_{3}$ ) with Pd-decorated stanene nanosheets using DFT was studied recently by Abassi [71]. Three different patterns for embedding $\mathrm{Pd}$ into the stanene ML was considered in the work before placement of the gas molecules on the Pd-decorated systems. It was shown that based on Mulliken charge analysis, the positive charge transfer occurred from the gas molecules to the Pd-decorated stanene systems and that pattern-III Pd-embedded stanene ML is the most energetically favourable one as shown in Figure 7 reproduced from this recent work.
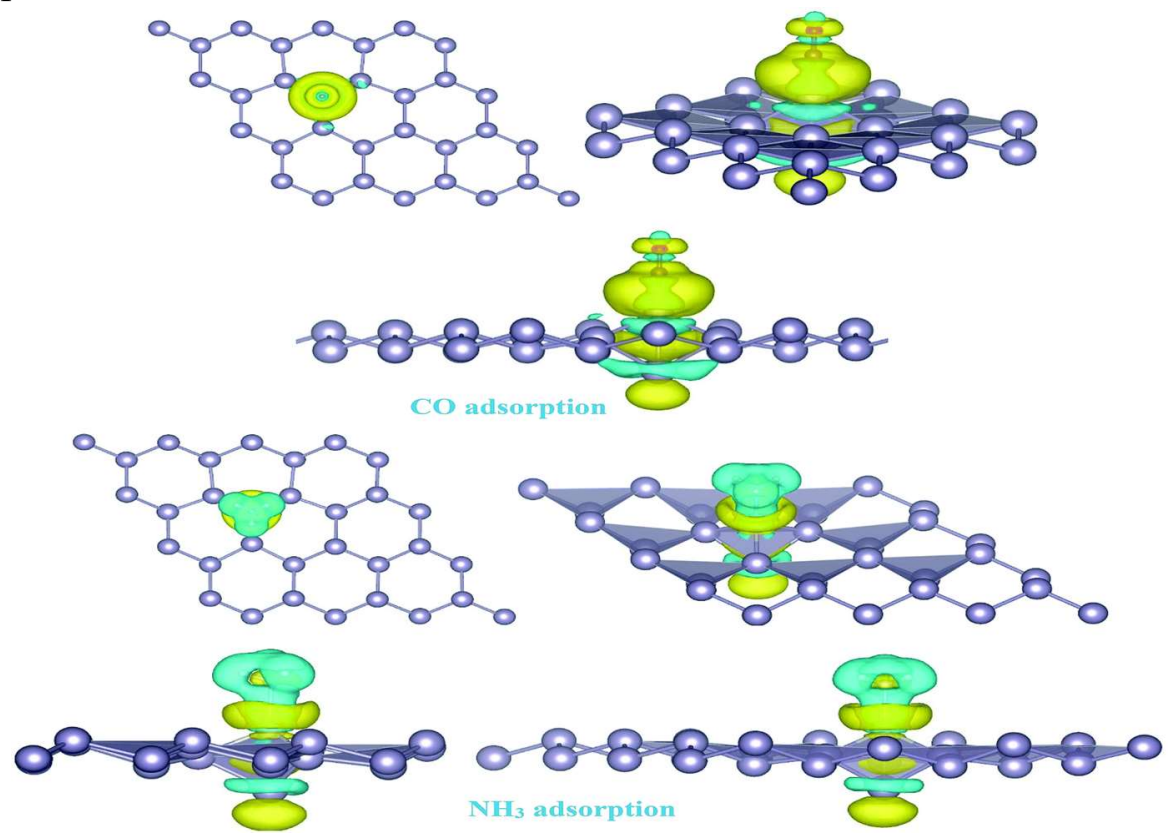

Figure 6.Isosurface plots of the electron charge density difference for pattern-III Pd-decorated stanene monolayers with adsorbed $\mathrm{CO}$ and $\mathrm{NH}_{3}$ molecules (isovalue is \pm 0.0002 a.u).[71] 


\section{Performance}

Sensitivity, selectivity, stability, response, and recovery time are the main parameters that govern the performance of a sensor. The sensor's response can be defined as the \% change in sensing property (with and without the presence of gas molecules) with respect to the original sensing property value of the sensor alone.

As one of the important parameters of gas sensors, sensitivity (S) can be defined as the measured signal change induced by per concentration unit of gases, and this value can be extracted from the slope of the curve based on the electrically measured parameter (resistance, voltage) vs the gas concentration in resistance based sensors.

Selectivity on the other hand is the capability of a gas sensor to respond to a specific group of gases or even to a certain gas in the presence of other gases. Usually, sensors are sensitive to more than one gas at the same time. Selectivity can be measured or estimated by comparing the effects of different gases on a sensor. Selectivity of the gas sensor is necessary for many applications and cross-sensitivity should be avoided.

The stability of a gas sensor characterizes the quality of a signal over a period of time and indicates the reliability of the senor measurements.

The response (time to reach $90 \%$ of response in the presence of gases) and recovery times (the time to return to $90 \%$ values in the absence of gases) are important key parameters of performance of a gas that are measured in the range of seconds to several tens of minutes.

So far, since all the gas sensing properties of pristine and doped stanene has been predicted from first principles using charge transfer, CDD, band structure analysis there are only qualitative results showing good, better, best or bad performance sensor ratings.

\section{Challenges and future outlook}

In conclusion, stanene the new young Gr cousin has great potential as a gas sensing device material in its pristine and doped forms as evidenced from the recent first principle calculations. This room temperature topological insulator is a novel $2 \mathrm{D}$ material that can overcome the deficiencies of Gr. with its excellent electronic properties.

Stanene stands out as the new 'gas sensing wonder' with its exceptional gas sensing performance. However there are quite a few challenges to be overcome. Although, it has been experimentally synthesized much work needs to done to reach the state of the art sophistication in large scale synthesis of stanene comparable to $\mathrm{Gr}$ and 2D MoS2. Device fabrication and testing are required to experimentally verify the theoretically predicted results. More intense research theoretically and practically is needed to form guidelines for stanene based sensors device fabrication that can make quantitative measurements and establish its superior gas sensing performance without doubt. Temperature and humidity also play important roles in the sensing performance of 2D materials and need to be investigated thoroughly. Repeatable, reliable, highly sensitive, selective and cost effective are the criteria that the future stanene based sensors need to address. 
References

[1] Zhang, Y.H., Chen, Y.B., Zhou, K.G., Liu, C.H., Zeng, J., Zhang, H.L. and Peng, Y. (2009) Improving gas sensing properties of graphene by introducing dopants and defects: a first-principles study. Nanotechnology, 20 (18).

[2] Seba S. Varghese, Sunil Lonkar, K.K. Singh, Sundaram Swaminathan, Ahmed Abdala, Recent advances in graphene based gas sensors, Sensors and Actuators B: Chemical, 218, 2015, 160-183.

[3] Ramanathan A.A. Defect Functionalization of $\mathrm{MoS}_{2}$ nanostructures as toxic gas sensors: A review. IOP Conf. Ser.: Mater. Sci. Eng. 2018, 305, 012001

[4] Kei Toda, Ryo Furue, Shinya Hayami. Recent progress in applications of graphene oxide for gas sensing: A review. Analytica Chimica Acta, 878, 2015, 43-53

[5] Yang S, Jiang C, and Wei S-H.Gas sensing in 2D materials. Appl. Phys. Rev. 2017 4, 021304

[6] Y. Jiang, L. Miao, G. Jiang, Y. Chen, X. Qi, X.-f. Jiang, H. Zhang, S. Wen. Sci. Rep., 5 (2015), 16372-16384

[7] H.-J. Li, L.-L. Wang, H. Zhang, Z.-R. Huang, B. Sun, X. Zhai, S.-C. Wen. Appl. Phys. Express, 2014, 7 Article 024301

[8] R. Wang, X. Li, Z. Wang, H. Zhang. Nano Energy, 2017, 34, 131-140

[9] Ramanathan A.A., Aqra M.W. and Al-Rawajfeh A.E Recent advances in 2D- nanopores for desalination. ECLE 2018, 16(4) 1217-1231.

[10] Yifei M. Liu, Christian Punckt, Michael A. Pope, Alan Gelperin, and Ilhan A. Aksay. Electrochemical Sensing of Nitric Oxide with Functionalized Graphene Electrodes. ACS Applied Materials \& Interfaces 2013, 5 (23), 12624-12630.

[11] Balendhran, S., Walia, S., Nili, H., Sriram, S. and Bhaskaran, M. (2015), Elemental Analogues of Graphene: Silicene, Germanene, Stanene, and Phosphorene. Small, 11: 640-652.

[12] D.J. Fisher. Elemental Graphene Analogues, Materials Research Foundations Vol 14, 2017, 384 Print ISBN 978-1-945291-30-2 ePDF ISBN 978-1-945291-31-9

DOI: $10.21741 / 9781945291319$

[13] Zongyu Huang, Huating Liu, Rong Hu, Hui Qiao, Huide Wang, Yundan Liu, Xiang Qi, Han Zhang. Structures, properties and application of 2D monoelemental materials (Xenes) as graphene analogues under defect engineering, Nano Today, 35, 2020, 100906

[14] Saxena, S. et al. Stanene: Atomically Thick Free-standing Layer of 2D Hexagonal Tin. Sci. Rep. 6, 31073; doi: 10.1038/srep31073 (2016).

[15] Novoselov K.S., Geim A.K., Morozov S.V., Jiang D., Zhang Y., Dubonos S.V., Grigorieva I.V., Firsov A.A. Electric field effect in atomically thin carbon films. Science. 2004;306:666-669.

[16] Max C. Lemme, “Current Status of Graphene Transistors”, Solid State Phenomena,Volumes 156-158, Pages 499-509, 2010. 
[17] Liu, C.-C., Jiang, H. \& Yao, Y. Low-energy effective Hamiltonian involving spin-orbit coupling in silicene and two-dimensional germanium and tin. Phys. Rev. B 84, 195430 (2011).

[18] Rita John, Benita Merlin, Optical properties of graphene, silicene, germanene, and stanene from IR to far UV - A first principles study, Journal of Physics and Chemistry of Solids, 110, 2017, 307-315.

[19] Elton J. G. Santos and Efthimios Kaxiras Nano Letters 201313 (3), 898-902

[20] Xiuhong Liu, Yu Wang, Feng Li and Yafei Li. Two-dimensional stanane: strain-tunable electronic structure, high carrier mobility, and pronounced light absorption. Phys. Chem. Chem. Phys., 2016, 18, 14638-14643

[21] S.S. Li, C.W. Zhang, Tunable electronic structures and magnetic properties in twodimensional stanene with hydrogenation, Mater. Chem. Phys. 173 (2016) 246-254.

[22] A.H. Castro Neto, F. Guinea, N.M.R. Peres, K.S. Novoselov, A.k. Geim, Rev. Mod. Phys. (2009) 81109-81162.

[23] Peng B, Zhang H, Shao H, Xu Y, Zhang X, Zhu H. Low lattice thermal conductivity of stanene. Sci Rep. 2016 Feb 3;6:20225.

[24] Chen S., Moore A.L., Cai W., Suk J.W., An J., Mishra C., Amos C., Magnuson C.W., Kang J., Shi L., et al. Raman measurements of thermal transport in suspended monolayer graphene of variable sizes in vacuum and gaseous environments. ACS

Nano. 2011;5:321-328.

[25] Bohayra Mortazavi, Obaidur Rahaman, Meysam Makaremi, Arezoo Dianat, Gianaurelio Cuniberti, Timon Rabczuk, First-principles investigation of mechanical properties of silicene, germanene and stanene, Physica E: Low-dimensional Systems and Nanostructures, 87, 2017, 228-232.

[26] Lee C., Wei X., Kysar J.W., Hone J. Measurement of the elastic properties and intrinsic strength of monolayer graphene. Science. 2008;321:385-388.

[27] Garg, P., Choudhuri, I., \& Pathak, B. (2017). Stanene based gas sensors: effect of spinorbit coupling. Physical chemistry chemical physics : PCCP, 19 46, 31325-31334 .

[28] Abbasi, A., \& Sardroodi, J.J. (2018). Density functional theory investigation of the interactions between the buckled stanene nanosheet and $\mathrm{XO} 2$ gases $(X=N, S, C)$. Computational and Theoretical Chemistry, 1125, 15-28.

[29] Zhang, A., Yang, H., Liu, Q., Li, W., \& Wang, Y. (2020). DFT insights into the adsorption properties of toxic gas molecules on pure and transition metal embedded stanene monolayers: Towards gas sensor devices. Synthetic Metals, 266, 116441.

[30] Chang, Y., Lin, J., Li, S., \& Liu, H. (2020). Adsorption of greenhouse gases (methane and carbon dioxide) on the pure and Pd-adsorbed stanene nanosheets: A theoretical study. Surfaces and Interfaces, 100878.

[31] Abbasi, A. (2019). Tuning the structural and electronic properties and chemical activities of stanene monolayers by embedding 4d Pd: a DFT study. RSC Advances, 9, 16069-16082. 
[32] Wang, D., Gao, H., Xiang, Y., \& Jiang, L. (2020). Tuning the structural and electronic properties of single-layer stanene through doping $4 \mathrm{~d}$ transition metals $(\mathrm{Mo}, \mathrm{Nb}, \mathrm{Rh}$ and Ru): A DFT study. Synthetic Metals, 264, 116399.

[33] P. Vogt, P.D. Padova, C. Quaresima, J. Avila, E. Frantzeskakis, M.C. Asensio, A. Resta, B. Ealet, G.L. Lay, Silicene: Compelling Experimental Evidence for Graphenelike Two-Dimensional Silicon. Phys. Rev. Lett. 108 (2012) 155501.

[34] A. Fleurence, R. Friedlein, T. Ozaki, H. Kawai, Y. Wang, Y. Yamada-Takamura, Phys. Rev. Lett. 108 (2012) 245501.

[35] H.-L. Guo, X.-F. Wang, Q.-Y. Qian, F.-B. Wang, X.-H. Xia, ACS Nano 3 (2009) 26532659.

[36] S. Cahangirov, M. Audiffred, P.Z. Tang, A. Iacomino, W. Duan, H.G. Merino, A. Rubio, Phys. Rev. B: Condens. Matter Mater. Phys. 88 (2013) 035432.

[37] Gao, J., Zhang, G. \& Zhang, YW. Exploring Ag(111) Substrate for Epitaxially Growing Monolayer Stanene: A First-Principles Study. Sci Rep 6, 29107 (2016).

[38] Dávila, M. E. \& Le Lay, G. Few layer epitaxial germanene: a novel two-dimensional Dirac material. Sci. Rep. 6, 20714 (2016).

[39] Li, L. et al. Buckled Germanene Formation on Pt(111). Adv. Mater. 26, 4820-4824 (2014).

[40] Liu, H., Gao, J. \& Zhao, J. From Boron Cluster to Two-Dimensional Boron Sheet on $\mathrm{Cu}(111)$ Surface: Growth Mechanism and Hole Formation. Sci. Rep. 3, 3238 (2013).

[41] Meng, L. et al. Buckled Silicene Formation on Ir(111). Nano Lett. 13, 685-690 (2013).

[42] F.-F. Zhu, W.-J. Chen, Y. Xu, C.-L. Gao, D.-D. Guan, C.-H. Liu, D. Qian, S.-C. Zhang, J.-F. Jia, Epitaxial growth of two-dimensional stanene. Nat. Mater. 14 (2015) 10201025.

[43] J. Qiao, X. Kong, Z.-X. Hu, F. Yang, W. Ji, High-mobility transport anisotropy and linear dichroism in few-layer black phosphorus, Nat. Commun. 5 (2014) 4475.

[44] H. Liu, A.T. Neal, Z. Zhu, Z. Luo, X. Xu, D. Tománek, P.D. Ye, Phosphorene: an unexplored 2D semiconductor with a high hole mobility, ACS Nano 8 (2014) 40334041.

[45] R. John, B. Merlin, Theoretical investigation of structural, electronic, and mechanical properties of two dimensional C, Si, Ge, Sn, Cryst. Struct. Theory Appl. 5 (2016) 43-55.

[46] Q. Yue, Z. Shao, S. Chang, and J. Li, Nanoscale Res. Lett. 8, 425 (2013).

[47] Tang Y, Chen W, Li C, Pan L, Dai X, Ma D Adsorption behavior of Co anchored on graphene sheets toward $\mathrm{NO}, \mathrm{SO}_{2}, \mathrm{NH}_{3}, \mathrm{CO}$ and $\mathrm{HCN}$ molecules. Applied Surface Science, 342, 191-199.

[48] L. J. Hubble, J. S. Cooper, A. Sosa-Pintos, H. Kiiveri, E. Chow, M. S.Webster, L. Wieczorek, and B. Raguse, ACS Comb. Sci. 17, 120-129 (2015).

[49] Y.Liu,J.Parisi,X.Sun,andY.Lei,J. Mater. Chem. A 2, 9919-9943 (2014).

[50] H. H. Pu, S. H. Rhim, M. Gajdardziksa-Josifovska, C. J. Hirschmugl, M.Weinert, and J. H. Chen, RSC Adv. 4, 47481-47487 (2014). 
[51] F. Schedin, A. K. Geim, S. V. Morozov, E. W. Hill, P. Blake, M. I. Katsnelson, and K. S. Novoselov, Nat. Mater. 6, 652-655 (2007).

[52] A. Singh, Md. A. Uddin, T. Sudarshan, and G. Koley, Small 10,1555-1565 (2014).

[53] J. Suehiro, G. Zhou, H. Imakiire, W. Ding, and M. Hara, Sens. Actuators, B108, 398403 (2005).

[54] C. C. Mayorga-Martinez, Z. Sofer, and M. Pumera, Angew. Chem., Int.Ed. 127, 1452514528 (2015).

[55] G. Korotcenkov and B. K. Cho, Sens. Actuators, B 188, 709-728 (2013).

[56] G. D. Francia, B. Alfano, and V. L. Ferrara, J. Sens. 2009, 1-18.

[57] F. K. Perkins, A. L. Friedman, E. Cobas, P. M. Campbell, G. G. Jernigan,and B. T. Jonker, Nano Lett. 13, 668-673 (2013).

[58] R. Arsat, M. Breedon, M. Shafiei, P. G. Spizziri, S. Gilje, R. B. Kaner, K. Kalantarzadeh, and W. Wlodarski, Chem. Phys. Lett. 467, 344-347 (2009).

[59] W. Yuan and G. Shi, J. Mater. Chem. A 1, 10078-10091 (2013).

[60] M. Qazi, T. Vogt, and G. Koley, Appl. Phys. Lett. 91, 233101 (2007).

[61] Z. Ling, C. Leach, and R. Freer, J. Am. Ceram. Soc. 23, 1881-1891 (2003).

[62] J. L. Johnson, A. Behnam, S. J. Pearton, and A. Ural, Adv. Mater. 22, 4877-4880 (2010).

[63] Y. Dan, Y. Lu, N. J. Kybert, Z. Luo, and A. T. C. Johnson, Nano Lett. 9,1472-1475 (2009).

[64] B. Zhan, C. Li, J. Yang, G. Jenkins, W. Huang, and X. Dong, Small 10, 4042-4065 (2014).

[65] G. Lu, S. Park, K. Yu, R. S. Ruoff, L. E. Ocola, D. Rosenmann, and J.Chen, ACS Nano 5, 1154-1164 (2011).

[66] C. C. Mayorga-Martinez, Z. Sofer, and M. Pumera, Angew. Chem., Int.Ed. 127, 1452514528 (2015).

[67] M. Modarresi et al. Effect of external strain on electronic structure of stanene. Comput. Mater. Sci. (2015)

[68] S. Kumar et al. Nano Lett. 2013, 13, 5, 1962-1968

[69] E. Bianco, S. Butler, S. Jiang, O. D. Restrepo, W. Windl and J. E. Goldberger, ACS Nano, 2013, 7, 4414-4421.

[70] Xiuhong Liu, Yu Wang, Feng Li and Yafei Li. Phys. Chem. Chem. Phys., 2016, 18, 14638--14643

[71] Amira Abassi. Tuning the structural and electronic properties and chemical activities of stanene monolayers by embedding 4d Pd: a DFT study, $\boldsymbol{R S C} \boldsymbol{A d \boldsymbol { v }}$. 2019,9, 16069-16082 
